\title{
Hydrodynamic Waves in Regions with Smooth Loss of Convexity of Isentropes. General Phenomenological Theory
}

\author{
Michael I. Tribelsky ${ }^{1}$, and Sergei I. Anisimovit ${ }^{2}$ \\ ${ }^{1}$ Department of Applied Physics, Faculty of Engineering, Fukui University, \\ Bunkyo 3-9-1, Fukui 910-8507, Japan \\ and Max-Planck-Institut für Physik komplexer Systeme, \\ Nöthnitzer Straße 38, D-01187 Dresden, Germany \\ ${ }^{2}$ Landau Institute for Theoretical Physics RAN, Kosygin St., Moscow 117334, Russia
}

(November 18, 2018)

\begin{abstract}
General phenomenological theory of hydrodynamic waves in regions with smooth loss of convexity of isentropes is developed based on the fact that for most media these regions in $p-V$ plane are anomalously small. Accordingly the waves are usually weak and can be described in the manner analogous to that for weak shock waves of compression. The corresponding generalized Burgers equation is derived and analyzed. The exact solution of the equation for steady shock waves of rarefaction is obtained and discusses.
\end{abstract}

PACS numbers: 47.40.-x, 43.25.+y, 52.35.Mw

Extremely great importance of the so-called elementary wave patterns, such as shock and rarefaction waves, in hydrodynamics has been known since the pioneering studies of Riemann [1] carried out in the beginning of 1860s. The importance is generally connected with (i) possibility to picture general fluids flows as nonlinear superposition of elementary waves, propagating as separate entities and (ii) reflection with the elementary waves the asymptotic behavior of general solutions of the set of hydrodynamic equations. For this reason a great deal of attention has been payed to study the elementary waves, see, e.g., the review article [2] and references therein. Nonetheless, a certain type of the elementary waves, namely shock waves of rarefaction and sonic waves [2], which admits general analytical consideration, has not been considered in such a manner. The gap is filled up in the present Letter.

It is well known that the sign of variation of pressure and density in a weak shock wave is associated with the sign of $\left(\partial^{2} V / \partial p^{2}\right)_{s}$. Usually the sign of the derivative is positive, and accordingly the shock wave is compressive. However, in some specific areas of $p-V$ plane, e.g., close to a critical point but still in a single-phase region, $\left(\partial^{2} V / \partial p^{2}\right)_{s}$ may become negative. The phenomenon is known as smooth loss of convexity of isentropes [2] and is quite general for many types of equations of states of real materials. Even the van der Waals equation possesses a region around the vapor dome, where $\left(\partial^{2} V / \partial p^{2}\right)_{s}<0$ [3]. In 1942 Bethe showed that all fluids having sufficiently large specific heats exhibit smooth loss of convexity of isentropes in the neighborhood of the saturated vapor line [⿰氵

The smooth loss of convexity of isentropes creates prerequisites for a shock wave of rarefaction (SWR) to come into being. Moreover, other types of anomalous wave structures, such as sonic shocks, whose Mach number(s) before the shock, after it, or both is (are) identically equal 1, may be observed in this region and its vicinity, see Refs. [2, 3] for more details. Bearing in mind all mentioned above, it is highly desirable to have a certain universal equation, which is not connected with any particular equation of state but neverthe- less can describe hydrodynamics waves in the discussed region. Despite the apparent importance of the problem and its long history, much to our surprise, nobody has payed attention to the following consequence of the fact that usually the region with the smooth loss of convexity of isentropes occupies a small domain in $p-V$ plane. The consequence is that the corresponding waves are always weak (otherwise the wave curves [5] go far away from the domain), and therefore the problem of the wave description is tractable within the framework of the macroscopic hydrodynamics in the manner analogous to that for weak shock waves of compression. To be precise we must stipulate that we do not consider any singularities related to the very vicinity to the critical point. In other words, it is implied the system is far enough from it to avoid any effect of the singularities.

In the present Letter the mentioned approach is applied to the problem. As a result the generalized Burgers equation, which describes hydrodynamic waves in the region, where the derivative $\left(\partial^{2} V / \partial p^{2}\right)_{s}$ is small and may change sign is derived. The equation is valid for both signs of the derivative and therefore describes all the variety of waves, which may exist in this region. As an example the equation is employed to study steady shock waves of rarefaction (SWR). The general exact solutions of the equation describing such shocks is obtained and analysed.

To begin with, let us consider the conventional Burgers equation, which governs a weak shock wave of compression. In the laboratory coordinate frame for a wave advancing in the negative direction of $x$-axis the equation reads [6]

$$
\frac{\partial p^{\prime}}{\partial t}-c_{1} \frac{\partial p^{\prime}}{\partial x}-\alpha p^{\prime} \frac{\partial p^{\prime}}{\partial x}=a c_{1}^{3} \frac{\partial^{2} p^{\prime}}{\partial x^{2}}
$$

where $p^{\prime}$ is the pressure variation, so that the pressure in the wave profile $p$ equals to $p_{1}+p^{\prime}$ (here and in what follows subscript 1 indicates the value of the corresponding quantity in the unperturbed medium, i.e., at $x \rightarrow-\infty$, while subscript 2 will stand for the value of the same quantity behind a wave, 
at $x \rightarrow \infty), c(p)$ is the velocity of sound,

$$
\alpha=\frac{c_{1}^{3}}{2 V^{2}}\left(\frac{\partial^{2} V}{\partial p^{2}}\right)_{s},
$$

$V$ denotes the specific volume $(V \equiv 1 / \rho), a$ is a dissipative constant [6]

$$
a \equiv \frac{1}{2 \rho_{1} c_{1}^{3}}\left[\left(\frac{4}{3} \eta+\zeta\right)+\kappa\left(\frac{1}{c_{v}}-\frac{1}{c_{p}}\right)\right],
$$

$\eta$ and $\zeta$ stand for the coefficients of viscosity, $\kappa$ for the thermal conductivity and $c_{v, p}$ for the corresponding specific heats.

Eq. (1) may be regarded as leading approximation to expansion of a certain more general nonlinear dissipative equation in powers of both weak nonlinearity and weak dissipation. It is important that the nonlinear term in this approximation is considered in non-dissipative limit. Dissipative corrections to it have additional smallness and may be neglected.

In our case $\left|\left(\partial^{2} V / \partial p^{2}\right)_{s}\right|$ is anomalously small (it even can vanish). Accordingly, the lowest nonlinearity $\alpha p^{\prime}\left(\partial p^{\prime} / \partial x\right)$ may not correspond to the leading nonlinear term any more. It means that higher order (in $p^{\prime}$ ) terms must be taken into account. On the other hand, the region, where $\left(\partial^{2} V / \partial p^{2}\right)_{s}<0$, is narrow, therefore the third derivative $\left(\partial^{3} V / \partial p^{3}\right)_{s}$ also has a certain smallness in this region. Only the fourth derivative $\left(\partial^{4} V / \partial p^{4}\right)_{s}$ does not have any smallness there. Thus, at small but finite $p^{\prime}$ terms $O\left(\left(p^{\prime}\right)^{2}\right), O\left(\left(p^{\prime}\right)^{3}\right)$ and $O\left(\left(p^{\prime}\right)^{4}\right)$ may yield contributions of the same order of magnitude and must be taken into account simultaneously. It is important that dissipative corrections to the nonlinear terms remain negligible and all such terms may be considered in the non-dissipative limit, in the same manner as it is for the conventional Burgers equation. All the above mentioned is related exclusively to nonlinear terms. Regarding the dissipative term on the right hand side of Eq. (1), there are no specific reasons for it to be small and no correction to this term is required.

To derive the desired nonlinear corrections to the left hand side of Eq. (1) let us consider an arbitrary adiabatic flow in the form of a traveling wave. Due to the adiabatic condition the profile of entropy of such a flow should not change, and since the state of the medium ahead of the shock front is spatially homogeneous with $s=$ const, we obtain that $s=$ const anywhere. Then, we may employ the general solution of the continuity and Euler equations valid for simple waves [6] and reduce these equations to the following single equation

$$
\frac{\partial p^{\prime}}{\partial t}+\left[v\left(p^{\prime}\right) \pm c\left(p^{\prime}\right)\right] \frac{\partial p^{\prime}}{\partial x}=0
$$

where the flow velocity $v$ is given by the expression

$$
v= \pm \int_{0}^{p^{\prime}} \frac{d p}{c(p) \rho(p)} .
$$

Sign plus in Eqs. (2)-(3) corresponds to the wave advancing in the positive $x$ direction, minus to negative. According to our choice of the direction of the wave propagation in what follows we take sign minus.

It is important to stress that under the above-specified conditions the reduction of the set of hydrodynamic equations to Eqs. (2) - (3) is an exact result valid for any nonlinear dependence $c(p)$ and $\rho(p)$, which may be obtained for the isentropic wave. However, if $p^{\prime}$ is small one can expand these functions about the point $p^{\prime}=0$. It is seen straightforwardly that if one truncates the expansion at zero term, it reduces Eqs. (2)-(3) to the linear acoustic equation. The truncation at the term of order $O\left(p^{\prime}\right)$ yields the left hand side of Eq. (1). Increasing the order of the truncation one can obtain the desired corrections to the conventional Burgers equation with any given accuracy.

Thus, the generalized Burgers equation must have the form

$$
\frac{\partial p^{\prime}}{\partial t}+u\left(p^{\prime}\right) \frac{\partial p^{\prime}}{\partial x}=c_{1}^{3} a \frac{\partial^{2} p^{\prime}}{\partial x^{2}},
$$

where $u$ stands for the velocity of a point in the wave profile. For our choice of the propagation direction $u\left(p^{\prime}\right)=v\left(p^{\prime}\right)-$ $c\left(p^{\prime}\right)$ [7].

To get the equation in the explicit form one must expand $u\left(p^{\prime}\right)$ in powers of small $p^{\prime}$ retaining the terms up to $O\left(\left(p^{\prime}\right)^{3}\right)$. However, before doing that it is worth analyzing some general features of Eq. (4).

First of all let us obtain the general expression for the velocity $v_{s}$ of a steady traveling shock, when $p^{\prime}(x, t) \rightarrow p^{\prime}(x+$ $\left.v_{s} t\right) \equiv p^{\prime}(\xi)$. In this case Eq. (4) may be easily integrated. Then, taking into account the boundary conditions $\left(p^{\prime} \rightarrow\right.$ $0, \partial p^{\prime} / \partial \xi \rightarrow 0$ at $\xi \rightarrow-\infty$ and $p^{\prime} \rightarrow p_{2}-p_{1}, \partial p^{\prime} / \partial \xi \rightarrow 0$ at $\xi \rightarrow \infty$ ), we arrive at the following expression for $v_{s}$

$$
v_{s}=-\frac{1}{p_{2}-p_{1}} \int_{0}^{p_{2}-p_{1}} u(p) d p \equiv-\langle u\rangle,
$$

where $\langle\ldots\rangle$ denotes average over $p^{\prime}$ [we remind that $u=v-$ $c<0$ see also Eq. (3)].

Let us show now that $v_{s}$ given by Eq. (5) for SWR satisfies the evolutionary conditions. The conditions say that in the comoving coordinate frame the velocity of the flow before the shock should be bigger than $c_{1}$, while the velocity behind the shock should be smaller than $c_{2}$, i.e., $M_{1}>1>M_{2}>0$, where $M_{1,2}$ stand for the corresponding Mach numbers. In the laboratory coordinate frame the medium before the shock is in the rest state with zero velocity. Accordingly, in the comoving coordinate frame the flow velocity here is $v_{s}$. Thus, the first evolutionary condition says $v_{s}>c_{1}$. Behind the shock the flow velocity in the laboratory frame is $v_{2}$. Consequently, the second condition yields $v_{s}+v_{2}<c_{2}$. Bearing in mind Eq. (5) both the conditions may be written as follows

$$
c_{1} \equiv-u_{1}<-\langle u\rangle<c_{2}-v_{2} \equiv-u_{2}
$$

Finally, taking into account that for a simple wave and the chosen direction of propagation the sign of derivative $d u / d p^{\prime}$ is opposite to that for $\left(\partial^{2} V / \partial p^{2}\right)_{s}$ [6], i.e., for negative $\left(\partial^{2} V / \partial p^{2}\right)_{s}$ function $-u\left(p^{\prime}\right)$ monotonically increases with 
decrease of $p^{\prime}$, and that for SWR $p_{2}$ is smaller than $p_{1}$, we reduce inequality (6) to evident.

Let us proceed with the derivation of the generalized Burgers equation. From all the above-mentioned it is clear that in the discussed region the derivative $\left(\partial^{2} V / \partial p^{2}\right)_{s}$ may be approximated as follows

$$
\left(\partial^{2} V / \partial p^{2}\right)_{s} \approx \frac{1}{\rho_{1}} \frac{1}{\left(\rho_{1} c_{1}^{2}\right)^{2}}\left[-\epsilon^{2}+\frac{\mu^{6}}{4}\left(\frac{p-p_{m}}{\rho_{1} c_{1}^{2}}\right)^{2}\right] .
$$

Here $p_{m}$ is the value of $p$, corresponding to the local minimum of the derivative, $\epsilon$ is a small dimensionless quantity $\mu=O(1)$ and power $\mu^{6}$ as well as the numerical coefficient are introduced for convenience of further notations.

According to Eq (7), $\left(\partial^{2} V / \partial p^{2}\right)_{s}<0$ at $p_{m}-\Delta<p<$ $p_{m}+\Delta$, where $\Delta=2 \rho_{1} c_{1}^{2} \epsilon / \mu^{3}=O(\epsilon)$. Then, expanding $u\left(p^{\prime}\right)$ in powers of $p^{\prime}$, taking into account that for the problem in question $p^{\prime}$ is of order $\epsilon$, or smaller and dropping terms higher than $O\left(\epsilon^{3}\right)$, after some calculations we reduce the general equation (4) to the form of Eq. (11), where term $\alpha p^{\prime}$ should be replaced by the following expression

$$
\begin{aligned}
\alpha p^{\prime} \rightarrow & \frac{\rho_{1}^{2} c_{1}^{3}}{2}\left[\left(\frac{\partial^{2} V}{\partial p^{2}}\right)_{s} p^{\prime}+\frac{1}{2}\left(\frac{\partial^{3} V}{\partial p^{3}}\right)_{s} p^{\prime 2}\right. \\
& \left.+\frac{1}{6}\left(\frac{\partial^{4} V}{\partial p^{4}}\right)_{s} p^{\prime 3}\right]
\end{aligned}
$$

and all derivatives $\left(\partial^{n} V / \partial p^{n}\right)_{s}$ are taken at $p=p_{1}$.

It is convenient to rewrite the equation in more universal dimensionless form. Let us introduce new variables

$$
y \equiv \frac{p^{\prime}}{\Delta} ; \quad \tau \equiv \frac{\epsilon^{6}}{\mu^{6} c_{1} a} t ; \quad \zeta \equiv \frac{\epsilon^{3}}{\mu^{3} c_{1}^{2} a}\left(x+c_{1} t\right) .
$$

In these variables involving expression (7) one can reduce the generalized Burgers equation to the following final form

$$
\frac{\partial y}{\partial \tau}-f(y) \frac{\partial y}{\partial \zeta}=\frac{\partial^{2} y}{\partial \zeta^{2}}
$$

where

$$
f(y) \equiv\left(z^{2}-1\right) y+z y^{2}+\frac{y^{3}}{3} ; z \equiv \frac{p_{1}-p_{m}}{\Delta} .
$$

In what follows we examine solutions of this equation, which correspond to SWR. In this case we should supplement Eq. (8) with the boundary conditions $\partial y / \partial \zeta \rightarrow 0$ at $\zeta \rightarrow \pm \infty ; y \rightarrow y_{1}=0$ at $\zeta \rightarrow-\infty ; y \rightarrow y_{2}=$ const $<0$ at $\zeta \rightarrow \infty$ and bear in mind that $y \leq 0 ;-1 \leq z \leq 1$. To study steady SWR it is convenient to introduce a traveling coordinate $\eta \equiv \zeta+\nu \tau$, where in accordance with Eq (5) the dimensionless velocity $\nu$ equals the following expression

$$
\nu=\frac{z^{2}-1}{2} y_{2}+\frac{z y_{2}^{2}}{3}+\frac{y_{2}^{3}}{12}
$$

Then, integration of Eq. (8) yields

$$
\begin{aligned}
& \eta=-12\left[-\frac{\ln |y|}{y_{2} y_{3} y_{4}}+\frac{\ln \left|y-y_{2}\right|}{y_{2}\left(y_{3}-y_{2}\right)\left(y_{4}-y_{2}\right)}\right. \\
& \left.+\frac{\ln \left|y-y_{3}\right|}{y_{3}\left(y_{3}-y_{4}\right)\left(y_{3}-y_{2}\right)}+\frac{\ln \left|y-y_{4}\right|}{y_{4}\left(y_{4}-y_{3}\right)\left(y_{4}-y_{2}\right)}\right],
\end{aligned}
$$

where $y_{3,4}$ are the roots of the equation

$$
y^{2}+\left(4 z+y_{2}\right) y+y_{2}^{2}+4 z y_{2}+6\left(z^{2}-1\right)=0
$$

Let us show that $y_{3,4}$ are always real and one of these root is smaller than $y_{2}$, while the other is bigger than $y_{1}$ (we remind that by definition $y_{1}=0$ ). In other words the left hand side of Eq. (11) is negative at $y_{2}<y<0$ and any possible values of $z$ and $y_{2}$. To prove it note that for SWR pressure $p_{2}$ must satisfy obvious conditions $p_{m}-\Delta \leq p_{2} \leq p_{1}$, which in the dimensionless variables are transformed into the following inequalities

$$
-(z+1) \leq y_{2} \leq 0 .
$$

Due to the fact that $y^{2}$ enters into Eq. 111) with a positive coefficient to prove negativeness of this polynomial at $y_{2}<$ $y<0$ it suffices to examine its values at the marginal points $y=y_{1,2}$. At $y=y_{1}=0$ we obtain

$$
y_{2}^{2}+4 z y_{2}+6\left(z^{2}-1\right) .
$$

For the same reason it is sufficient to inspect the values of polynomial (12) at the marginal values of $y_{2}$, namely at $y_{2}=$ $-(z+1), y_{2}=0$. It is straightforward to see that the marginal values of Eq. (12) are negative at $|z|<1$. Negativeness of polynomial (11) at $y=y_{2}$ is proved in the same manner. The proved relative position of points $y_{1,2,3,4}$ guarantees that the derivative $d y / d \eta$ is negative at $y_{2}<y<y_{1}$ for any possible values of $z$ and $y_{2}$, i.e., the profile of the steady SWR is a monotonically decreasing function of $\eta$.

To end this Letter we present several particular versions of general solution (10), when the dependence $y(\eta)$ may be obtained explicitly.

(i) $z=1 y_{2}=-2$, which corresponds the maximal possible amplitude of SWR $\left(p_{1}=p_{m}+\Delta, p_{2}=p_{m}-\Delta\right)$.

$$
\eta=-\frac{3}{2} \ln \left|\frac{2+y}{y}\right|-\frac{3}{2 \sqrt{5}} \ln \frac{y+1+\sqrt{5}}{\sqrt{5}-1-y} .
$$

(ii) $z=1\left|y_{2}\right| \ll 1 p_{1}=p_{m}+\Delta, p_{1}-p_{2} \ll \Delta$. In this case the leading approximation in small $\left|y_{2}\right|$ yields

$$
y=y_{2} \sqrt{\frac{1+\tanh \left(y_{2}^{2} \eta / 3\right)}{2}}
$$

(iii) $z=-\left(1+y_{2}\right),\left|y_{2}\right| \ll 1\left[p_{1}=p_{m}-\Delta-\left(p_{1}-\right.\right.$ $\left.\left.p_{2}\right), p_{1}-p_{2} \ll \Delta\right]$. The leading approximation in $\left|y_{2}\right|$ in this case results in the following profile

$$
y=y_{2}\left[1-\sqrt{\frac{1-\tanh \left(y_{2}^{2} \eta / 3\right)}{2}}\right]
$$

Note, that while in the present Letter only steady solutions of Eq. (8)-(9) corresponding to SWR are discussed, the equation itself describes much more broad spectrum of problems 
of steady and non-steady flows, including such very interesting issues, as sonic waves, evolution of arbitrary initial profiles, collision of shocks, etc. The corresponding study is in progress and results will be reported elsewhere.

One of the authors (M.I.T.) is grateful to Peter Fulde for invitation to MPI-PKS, which provides the opportunity to complete the present study, and to the entire staff of the Institute for kind hospitality. This work was supported by the Grantin-Aid for Scientific Research (No. 11837006) from the Ministry of Education, Culture, Sports, Science and Technology (Japan).
* Electronic address: tribel@ scroll.apphy.fukui-u.ac.jp

$\dagger \quad$ Electronic address: anisimov@itp.ac.ru

[1] B. Riemann Collected Works of Bernhard Riemann, edited by H.Weber (Dover, New York, 1953).

[2] R. Menikoff, and B. J. Plohr, Rev. Mod. Phys. 61, 75 (1989).

[3] M. Cramer, and R. Sen, Phys. Fluids 30, 377 (1987).

[4] H. Bethe, The theory of shock waves for an arbitrary equation of state (Clearinghouse for Federal Scientific and Technical Information, U. S. Department of Commerce, Washington D.C.), Report No. PB-32189.

[5] A curve, which consists of states connecting initial and final states by a given wave solution.

[6] L. D. Landau, and E. M. Lifshitz, Fluid Mechanics - 2nd ed. (Butterworth-Heinemann, Oxford, 1995).

[7] For a wave traveling in the opposite direction we would have $u\left(p^{\prime}\right)=v\left(p^{\prime}\right)+c\left(p^{\prime}\right)$. 